\title{
Synthesis of 2,8-diamino-5-hydroxy-4H,10H- pyrano[2,3-f]chromene-3,9-dicarbonitriles
}

Victor V. Dotsenko ${ }^{1}$, Ivan V. Didenko ${ }^{1}$, Gennady D. Krapivin ${ }^{2}$, Nikolay A. Aksenov $^{3}$, Inna V. Aksenova ${ }^{3}$, Sergey G. Krivokolysko ${ }^{4,5}$

${ }^{1}$ Kuban State University, 149 Stavropolskaya Str., 350040 Krasnodar, Russian Federation

${ }^{2}$ Kuban State Technological University, 2 Moskovskaya str., 350072 Krasnodar, Russian Federation

${ }^{3}$ Department of Chemistry, North Caucasus Federal University, la Pushkin Str., 355009 Stavropol, Russian Federation

${ }^{4}$ ChemEx Lab, Vladimir Dal' Lugansk National University, 20A Molodezhny, 91034 Lugansk, Russian Federation

${ }^{5}$ Lugansk State Medical University, 1-g 50 years of Lugansk Defence, 91045

Lugansk, Russian Federation

Abstract: The reactions of phloroglucinol with $\alpha, \beta$-unsaturated dinitriles (or malononitrile and carbonyl compounds) lead to the formation of hitherto unknown 2,8-diamino-5-hydroxy-4H,10H-pyrano[2,3-f]chromene-3,9-dicarbonitriles. The reaction conditions and the structure of the products were studied in details.

Keywords: phloroglucinol, malononitrile, isatin, NMR studies, X-ray studies, 2amino-4H-pyran-3-carbonitrile, pyrano[2,3-f]chromene.

The great interest in the chemistry of 2 -amino- $4 H$-chromenes, especially those bearing the nitrile group at the 3-position is essentially inspired by the availability of this class of compounds - on the one hand, and biological activity of many 2-amino- $4 \mathrm{H}$-chromenes - on the other. The chemistry of 2-amino- $4 \mathrm{H}$ chromenes have been reviewed [1-3]. As it was reported in the literature [4], the reaction of benzaldehydes and malononitrile with phloroglucinol taken in the ratio $1: 1: 1$ leads to the formation of 2-amino-3-cyano-4H-chromenes $\mathbf{1}$ :<smiles>CC(C)(N)C(CC#N)C(=O)O</smiles>

Since phloroglucinol could be considered as a tautomeric form of cyclohexan-1,3,5-trione, one may suggest that phloroglucinol may react with activated nitriles to form three- or tetracyclic pyran compounds. We decided to study the reaction in order to obtain products with the proposed structures $\mathbf{2 - 4}$ : 
<smiles>N#CC1=C(N)Oc2cc3c(c(O)c2C1[Al])C([Al])C(C#N)=C(N)O3</smiles><smiles>N#CC1=C(N)Oc2c(cc(O)c3c2C([Al])C(C#N)=C(N)O3)O1</smiles><smiles></smiles>

It was found that when phloroglucinol reacted with malononitrile and aromatic aldehydes taken in a ratio of $1: 2: 2$, or with arylmethylene malononitriles in a ratio of $1: 2$, both in the presence of catalytic amounts of morpholine in boiling EtOH - the same products with the possible pyranochromene structures $\mathbf{2}$ and $\mathbf{3}$ were formed. The choice between two possible structures - linear $\mathbf{2}$ and angular one $\mathbf{3}$ - was made in favor of the angular structure $\mathbf{3}$ on the basis of NMR spectroscopy. Isatin as an active carbonyl compound was reacted with malononitrile and phloroglucinol in hot $\mathrm{EtOH}$ to afford spirocyclic pyranochromene $\mathbf{5}$ in $47 \%$ yield.<smiles>CCOC(C)C(C)C(C)C=O</smiles><smiles>CCOCCO</smiles><smiles>N#CC1=C(N)Oc2ccccc2C12C(C#N)=C(N)Oc1c2ccc2c1C1(C(=O)Nc3ccccc31)C(=O)N2</smiles>

$\mathrm{Ar}=4-\mathrm{MeOC}_{6} \mathrm{H}_{4}, 3$-pyridyl

Thus, the ${ }^{1} \mathrm{H}$ NMR spectrum of crude compound 3a (obtained from the reaction of 2 eq. 4-(methoxybenzylidene)malononitrile with phloroglucinol) revealed the doubling of both the aryl protons signals and chromene proton H-4. Such a spectrum is impossible for symmetric linear structures like pyranochromene 2. Figure 1 shows a fragment of the ${ }^{1} \mathrm{H}$ NMR spectrum of crude compound 3a. Thus, the presence of two different signals for $\mathrm{H}-4$ protons of $4 \mathrm{H}-$ pyran ring at $\delta$ $4,45 \mathrm{ppm}$ and $4.68 \mathrm{ppm}$ clearly indicates their non-equivalence, and thus confirms the asymmetric structure of the product. It should be noted that only one signal corresponding to $\mathrm{OH}$ group was observed at $\delta 10,00 \mathrm{ppm}$, and one signal of an aryl 
proton only was observed at $\delta 6,28 \mathrm{ppm}$, whereas the signals of the aromatic substituents are doubled. Pyridine-3-carbaldehyde as well as it's condensation product with malononitrile were found to react the same way. Thus, compound $\mathbf{3 b}$ was obtained in $70 \%$ yield when phloroglucinol was reacted with two equivalents of malononitrile and pyridine-3-carbaldehyde.

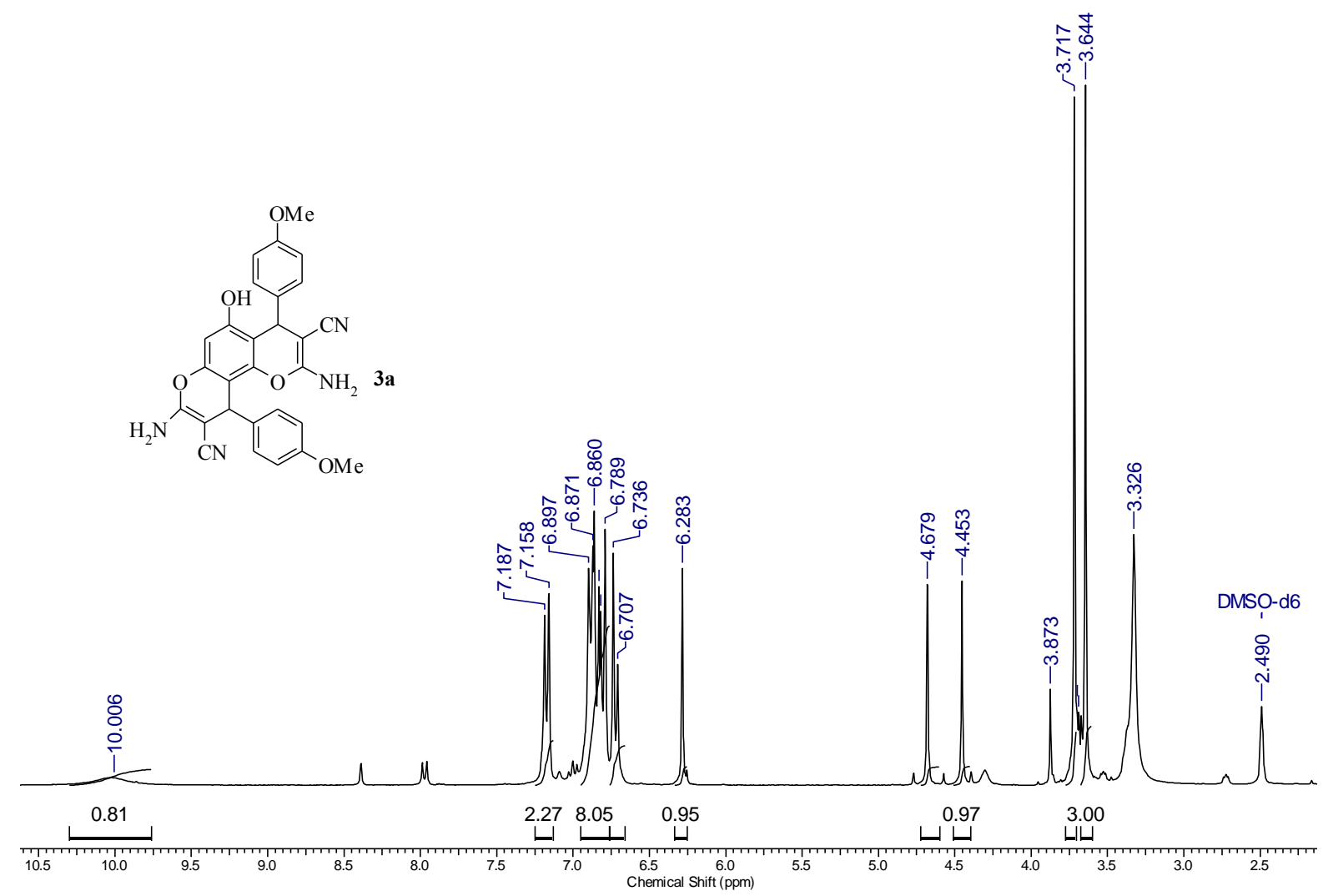

Fig. 1. The fragment of the ${ }^{1} \mathrm{H}$ NMR spectrum of crude $\mathbf{3 a}$.

We failed to obtain tetracyclic compounds $\mathbf{4}$ from the reaction of phloroglucinol with malononitrile and aromatic aldehydes taken in a ratio of $1: 3$ : 3 under the same conditions. In this case, the sole products isolated were arylmethylene malononitriles $\mathbf{6}$.

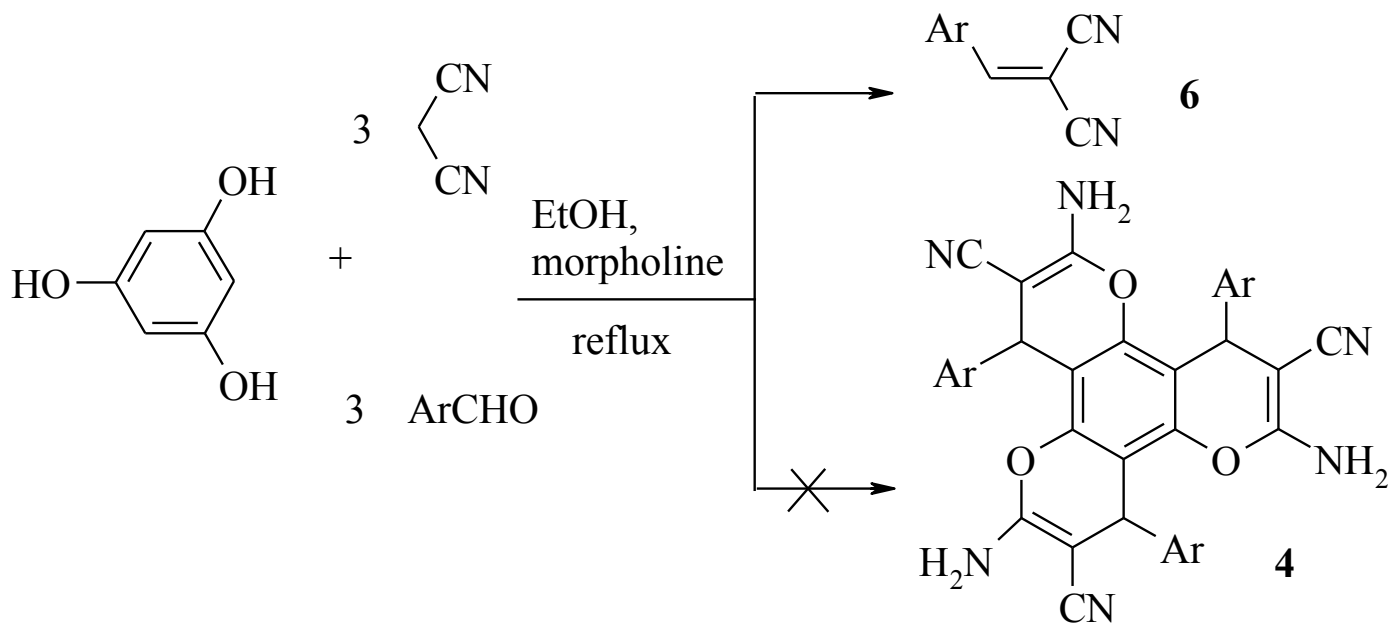

$6 \mathrm{Ar}=\mathrm{Ph}, 4-\mathrm{AcNHC}_{6} \mathrm{H}_{4}, 2$-furyl. 
Aliphatic aldehydes do not react under these conditions to give pyranochromenes. The structure of the obtained compounds was confirmed by means of NMR, FTIR and LCMS. The structure of the condensation product $\mathbf{6 b}$ was studied by X-ray analysis (Figure 2).

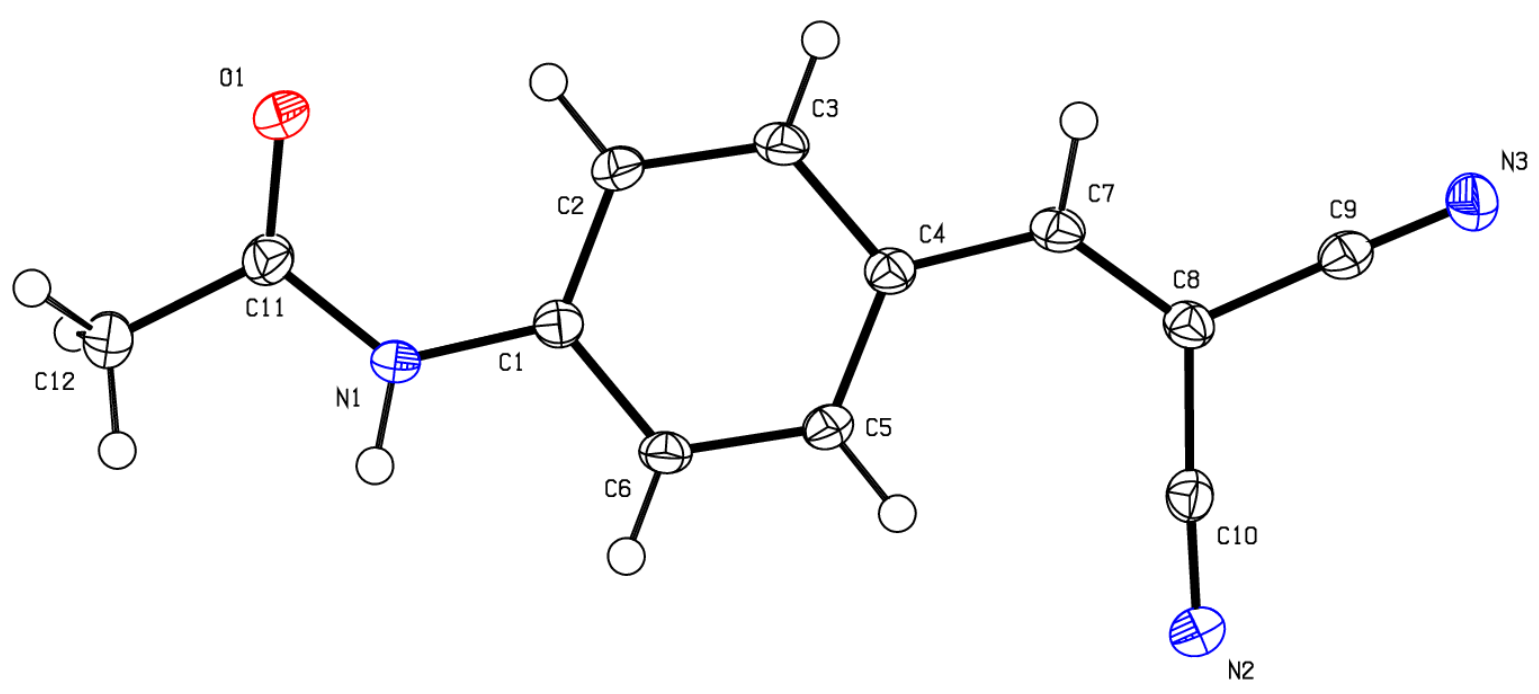

Fig. 2. The structure of $4-\mathrm{AcNHC}_{6} \mathrm{H}_{4} \mathrm{CH}=\mathrm{C}(\mathrm{CN})_{2}$.

\section{Typical experimental procedure}

Synthesis of 2,8-diamino-5-hydroxy-4,10-di(3-pyridyl)-4H,10H-pyrano[2,3f]chromen-3,9-dicarbonitrile.<smiles>N#CC1=C(N)Oc2cc(O)c3c(c2C1c1cccnc1)C(c1cccnc1)C(C#N)=C(N)O3</smiles>

A round bottom $50 \mathrm{~mL}$ flask was charged with malononitrile $(0.4 \mathrm{~g}, 0.00606 \mathrm{~mol})$, pyridine-3-carbaldehyde $(0.57 \mathrm{ml}, 0.00606 \mathrm{~mol})$ and $\mathrm{EtOH}(10 \mathrm{~mL})$. Then 2 drops of morpholine were added, and, after 5 minutes $-0.38 \mathrm{~g}(0.00303 \mathrm{~mol})$ of phloroglucinol. The reaction mixture was refluxed for $6 \mathrm{~h}$. Upon cooling, the beige solid precipitated. The precipitate was filtered off and washed with EtOH and $\mathrm{BuOH}$ to give $0.96 \mathrm{~g}(70 \%)$ of $\mathbf{3 b}$.

\section{References}

1. Yu A Sharanin, M P Goncharenko, V P Litvinov. Reactions of carbonyl compounds with a,b-unsaturated nitriles as a convenient pathway to carboand heterocycles// Russian Chemical Reviews - 1998, Vol. 67. - No. 5. pp. $393-422$. 
2. Ahmed M. El-Agrody and Tarek H. Afifi. The reactivity of 8hydroxyquinoline and its derivatives toward $\alpha$-cyanocinnamonitriles and ethyl $\alpha$-cyanocinnamates: synthesis, reactions, and applications of $4 \mathrm{H}-$ pyrano[3,2-h]quinoline derivatives // Heterocycles - 2014, Vol. 89 - №. 7. pp. 1557-1584.

3. Yuri M. Litvinov and Anatoliy M. Shestopalov. Synthesis, Structure, Chemical Reactivity, and Practical Significance of 2-Amino-4H-pyrans. In Advances in Heterocyclic Chemistry (ed. A. R. Katritzky), 2011, Vol. 103, p. 175

4. I. B. Masesane, S. O. Mihigo. Efficient and green preparation of 2-amino$4 \mathrm{H}$-chromenes by a room-temperature, $\mathrm{Na}_{2} \mathrm{CO}_{3}$-catalyzed, three-component reaction of malononitrile, benzaldehydes, and phloroglucinol or resorcinol in aqueous medium // Synthetic Communications - 2015, Vol. 45 - №. 13. - P. $1546-1551$. 